\title{
On the increasing solutions of the translation equation
}

\author{
by JANUSz BRZDȨK (Rzeszów)
}

\begin{abstract}
Let $M$ be a non-empty set endowed with a dense linear order without the smallest and greatest elements. Let $(G,+)$ be a group which has a non-trivial uniquely divisible subgroup. There are given conditions under which every solution $F: M \times G \rightarrow M$ of the translation equation is of the form $F(a, x)=f^{-1}(f(a)+c(x))$ for $a \in M, x \in G$ with some non-trivial additive function $c: G \rightarrow \mathbb{R}$ and a strictly increasing function $f: M \rightarrow \mathbb{R}$ such that $f(M)+c(G) \subset f(M)$. In particular, a problem of J. Tabor is solved.
\end{abstract}

This paper is motivated by the following problem raised by J. Tabor during his talk at the Mathematics Department of the Pedagogical University in Rzeszów:

Let $M$ be a non-empty set endowed with a dense linear order $\leq$ without the smallest and greatest elements and let $\mathbb{R}$ stand for the set of all reals. Find conditions such that a function $F: M \times \mathbb{R} \rightarrow M$ satisfies them and the translation equation

$$
F(F(a, x), y)=F(a, x+y)
$$

if and only if there exist an additive function $c: \mathbb{R} \rightarrow \mathbb{R}$, a non-empty set $T \subset \mathbb{R}$, and a strictly increasing function $f$ mapping $M$ onto the set $T+c(\mathbb{R})$ such that $c(\mathbb{R})$ is dense in $\mathbb{R}$ and

$$
F(a, x)=f^{-1}(f(a)+c(x))
$$

for $x \in \mathbb{R}, a \in M$.

The problem is connected with some iteration groups. For the details we refer to [4].

We present a solution of the problem. We also give some results concerning monotonic solutions of equation (1). Such solutions of the translation equation have already been studied e.g. in [1]-[3]. However, the literature devoted to them is not very vast.

1991 Mathematics Subject Classification: Primary 39B52.

Key words and phrases: translation equation, linear order, increasing function, additive function. 
Throughout this paper $\mathbb{N}, \mathbb{Z}, \mathbb{Q}$ stand for the sets of all positive integers, all integers, and rationals, respectively. $(G,+)$ denotes a group (not necessarily commutative) and $H$ is a non-trivial uniquely divisible subgroup of $G$, unless explicitly stated otherwise.

Let us start with the following

Lemma 1. Let $x_{0} \in H \backslash\{0\}$ and $b \in M$. Suppose that a function $F$ : $M \times G \rightarrow M$ satisfies the following two conditions:

(3) the set $\left\{F\left(b, p x_{0}\right): p \in \mathbb{Q}\right\}$ is dense in $M$,

(4) $F\left(b, p x_{0}\right)<F\left(b, q x_{0}\right)$ for every $p, q \in \mathbb{Q}, p<q$.

Then, for each $t \in \mathbb{R}, t>0(t<0$ respectively $)$, the function $f_{t}: M \rightarrow \mathbb{R}$ given by

$$
f_{t}(a)=t \lim _{n \rightarrow \infty} q_{n}^{a} \quad \text { for } a \in M,
$$

where $\left\{q_{n}^{a}: n \in \mathbb{N}\right\} \subset \mathbb{Q}$ is any strictly increasing sequence such that $a=$ $\lim _{n \rightarrow \infty} F\left(b, q_{n}^{a} x_{0}\right)$ (with respect to the order in $M$ ), is strictly increasing (decreasing resp.).

Proof. Fix $t \in \mathbb{R}, t>0$ (in the case $t<0$ the proof is analogous). First, we prove that $f_{t}$ is well defined.

Fix $a \in M$. Since $M$ is without a greatest element, by (3) there is $p \in \mathbb{Q}$ with $a<F\left(b, p x_{0}\right)$. Thus, according to (4), there is a real number $s:=\sup \left\{q \in \mathbb{Q}: F\left(b, q x_{0}\right)<a\right\}<p$. Let $\left\{q_{n}^{a}: n \in \mathbb{N}\right\} \subset \mathbb{Q}$ be a strictly increasing sequence with $s=\lim _{n \rightarrow \infty} q_{n}^{a}$. Then $\left\{F\left(b, q_{n}^{a} x_{0}\right): n \in \mathbb{N}\right\}$ is a strictly increasing sequence in $M$. Take $d \in M$ with $d<a$. There is $r \in \mathbb{Q}$ with $d<F\left(b, r x_{0}\right)<a$. Further, there exists $m \in \mathbb{N}$ with $r<q_{m}^{a}$. Consequently, $d<F\left(b, r x_{0}\right)<F\left(b, q_{n}^{a} x_{0}\right)<a$ for every $n \in \mathbb{N}$ with $n>m$. This means that $a=\lim _{n \rightarrow \infty} F\left(b, q_{n}^{a} x_{0}\right)$.

Next, suppose that $\left\{p_{n}^{a}: n \in \mathbb{N}\right\} \subset \mathbb{Q}$ is also a strictly increasing sequence such that $a=\lim _{n \rightarrow \infty} F\left(b, p_{n}^{a} x_{0}\right)$. Then, for every $n \in \mathbb{N}$, there are $k, m \in \mathbb{N}$ with $F\left(b, q_{n}^{a} x_{0}\right)<F\left(b, p_{k}^{a} x_{0}\right)$ and $F\left(b, p_{n}^{a} x_{0}\right)<F\left(b, q_{m}^{a} x_{0}\right)$. Hence (4) $i m p l i e s \lim _{n \rightarrow \infty} q_{n}^{a}=\lim _{n \rightarrow \infty} p_{n}^{a}$. In this way we have proved that the definition of $f_{t}$ is correct.

To complete the proof, fix $a, d \in M, a<d$. Let $\left\{q_{n}^{a}: n \in \mathbb{N}\right\},\left\{q_{n}^{d}: n \in\right.$ $\mathbb{N}\} \subset \mathbb{Q}$ be strictly increasing sequences with $a=\lim _{n \rightarrow \infty} F\left(b, q_{n}^{a} x_{0}\right)$ and $d=\lim _{n \rightarrow \infty} F\left(b, q_{n}^{d} x_{0}\right)$. Since $a<d$, there is $n_{0} \in \mathbb{N}$ such that

$$
F\left(b, q_{k}^{a} x_{0}\right)<F\left(b, q_{n}^{d} x_{0}\right) \quad \text { for every } k, n \in \mathbb{N}, n>n_{0} .
$$

Thus, by (4), $q_{k}^{a}<q_{n}^{d}$ for every $k, n \in \mathbb{N}, n>n_{0}$, which means that $\lim _{n \rightarrow \infty} q_{n}^{a}<\lim _{n \rightarrow \infty} q_{n}^{d}$. Hence $f_{t}(a)<f_{t}(d)$. This ends the proof.

Lemma 2. Let $a, b \in M$ and $x_{0} \in H \backslash\{0\}$. Suppose that $F: M \times G \rightarrow M$ is a function and $\left\{q_{n}^{a}: n \in \mathbb{N}\right\} \subset \mathbb{Q}$ is a strictly increasing sequence such 
that $a=\lim _{n \rightarrow \infty} F\left(b, q_{n}^{a} x_{0}\right)$, (3) and (4) hold,

(6) the set $\{F(d, x): d \in M\}$ is dense in $M$ for every $x \in G$,

and

(7) $F(d, x)<F(c, x) \quad$ for every $d, c \in M, d<c, x \in G$.

Then $F(a, x)=\lim _{n \rightarrow \infty} F\left(F\left(b, q_{n}^{a} x_{0}\right), x\right)$ for every $x \in G$.

Proof. Fix $x \in G$. Since, according to (3), the set $\left\{F\left(b, p x_{0}\right): p \in \mathbb{Q}\right\}$ is dense in $M$, so is the set $\left\{F\left(F\left(b, p x_{0}\right), x\right): p \in \mathbb{Q}\right\}$ by (6) and (7). Moreover, by (7), for every $p \in \mathbb{Q}$ with $F\left(F\left(b, p x_{0}\right), x\right)<F(a, x)$, there exists $m \in \mathbb{N}$ such that

$$
F\left(F\left(b, p x_{0}\right), x\right)<F\left(F\left(b, q_{n}^{a} x_{0}\right), x\right)<F(a, x) \quad \text { for } n \in \mathbb{N}, n>m,
$$

because $a=\lim _{n \rightarrow \infty} F\left(b, q_{n}^{a} x_{0}\right)$ and, by (4) and (7), the sequence $\left\{F\left(F\left(b, q_{n}^{a} x_{0}\right), x\right): n \in \mathbb{N}\right\}$ is strictly increasing. This yields the assertion.

Lemma 3. Let $b, d \in M,\left\{a_{k}: k \in \mathbb{N}\right\} \subset M, x_{0} \in H \backslash\{0\}, F: M \times G \rightarrow$ $M$ and let $\left\{p_{n}: n \in \mathbb{N}\right\},\left\{q_{n}: n \in \mathbb{N}\right\} \subset \mathbb{Q}$ be bounded strictly increasing sequences such that $a_{k}=\lim _{n \rightarrow \infty} F\left(b,\left(q_{k}+p_{n}\right) x_{0}\right)$ for $k \in \mathbb{N}$ and $d=$ $\lim _{k \rightarrow \infty} a_{k}$. Suppose that condition (4) holds. Then $d=\lim _{n \rightarrow \infty} F\left(b,\left(q_{n}+\right.\right.$ $\left.\left.p_{n}\right) x_{0}\right)$.

Proof. Fix $k \in \mathbb{N}$. Then there exists $n_{0} \in \mathbb{N}, n_{0}>k$, with

$$
\begin{aligned}
q_{k}+p_{j} & <q_{k+1}+p_{j}<q_{k+1}+\lim _{n \rightarrow \infty} p_{n}<q_{m}+p_{m} \\
& <q_{m}+p_{m+j} \quad \text { for } j, m \in \mathbb{N}, m>n_{0},
\end{aligned}
$$

because the sequences $\left\{p_{n}: n \in \mathbb{N}\right\}$ and $\left\{q_{n}: n \in \mathbb{N}\right\}$ are strictly increasing and bounded. Thus, according to (4), $a_{k} \leq a_{k+1}$ and

$$
F\left(b,\left(q_{k}+p_{n}\right) x_{0}\right)<F\left(b,\left(q_{m}+p_{m}\right) x_{0}\right)<a_{m} \quad \text { for } n, m \in \mathbb{N}, m>n_{0} .
$$

In this way we have proved that the sequence $\left\{a_{k}: k \in \mathbb{N}\right\}$ is increasing and, for each $k \in \mathbb{N}$, there exists $m \in \mathbb{N}$ with

$$
a_{k} \leq F\left(b,\left(q_{m}+p_{m}\right) x_{0}\right) \leq a_{m},
$$

which yields the assertion.

Lemma 4. Let $x_{0} \in H \backslash\{0\}, z \in G, b \in M$ and let $F: M \times G \rightarrow M$ be a function satisfying

$$
\begin{aligned}
F(F(b, x), y) & =F(b, x+y) & & \text { for } x, y \in G, \\
F(b, x+y) & =F(b, y+x) & & \text { for } x, y \in G
\end{aligned}
$$

and conditions (3), (4), (6), (7). Suppose that $\left\{q_{n}^{z}: n \in \mathbb{N}\right\} \subset \mathbb{Q}$ is a strictly increasing sequence such that $F(b, z)=\lim _{n \rightarrow \infty} F\left(b, q_{n}^{z} x_{0}\right)$. Then

$$
F(a, z)=\lim _{n \rightarrow \infty} F\left(a, q_{n}^{z} x_{0}\right) \quad \text { for every } a \in M .
$$


Proof. Fix $a \in M$. Then, by (3) and (4), there is a strictly increasing bounded sequence $\left\{q_{n}^{a}: n \in \mathbb{N}\right\} \subset \mathbb{Q}$ such that $a=\lim _{n \rightarrow \infty} F\left(b, q_{n}^{a} x_{0}\right)$. According to Lemma 2 and conditions $\left(1^{\prime}\right)$ and (8),

$$
\begin{aligned}
F(a, z) & =\lim _{n \rightarrow \infty} F\left(F\left(b, q_{n}^{a} x_{0}\right), z\right)=\lim _{n \rightarrow \infty} F\left(F(b, z), q_{n}^{z} x_{0}\right) \\
& =\lim _{n \rightarrow \infty}\left(\lim _{k \rightarrow \infty} F\left(F\left(b, q_{k}^{z} x_{0}\right), q_{n}^{a} x_{0}\right)\right) \\
& =\lim _{n \rightarrow \infty}\left(\lim _{k \rightarrow \infty} F\left(b,\left(q_{n}^{a}+q_{k}^{z}\right) x_{0}\right)\right)=: I_{1}
\end{aligned}
$$

and

$$
\begin{aligned}
\lim _{k \rightarrow \infty} F\left(a, q_{k}^{z} x_{0}\right) & =\lim _{k \rightarrow \infty}\left(\lim _{n \rightarrow \infty} F\left(F\left(b, q_{n}^{a} x_{0}\right), q_{k}^{z} x_{0}\right)\right) \\
& =\lim _{k \rightarrow \infty}\left(\lim _{n \rightarrow \infty} F\left(b,\left(q_{n}^{a}+q_{k}^{z}\right) x_{0}\right)\right)=: I_{2} .
\end{aligned}
$$

Since, by Lemma $3, I_{1}=I_{2}$, we obtain the statement.

LemMA 5. Let $b \in M, x_{0} \in H \backslash\{0\}$ and let $F: M \times G \rightarrow M$ be a function satisfying conditions $\left(1^{\prime}\right),(3),(4)$, and (6)-(8). Then, for each $t \in \mathbb{R}, t>0$ $(t<0$ resp. $)$, the function $c_{t}: G \rightarrow \mathbb{R}, c_{t}(x)=f_{t}(F(b, x))$ for $x \in G$, is additive, $c_{t}\left(x_{0}\right)>0\left(c_{t}\left(x_{0}\right)<0\right.$ resp. $)$, and

$$
F(a, x)=f_{t}^{-1}\left(f_{t}(a)+c_{t}(x)\right) \quad \text { for } a \in M, x \in G,
$$

where the function $f_{t}: M \rightarrow \mathbb{R}$ is given by (5).

Proof. Fix $a \in M, x, y \in G, t \in \mathbb{R}, t \neq 0$, and strictly increasing bounded sequences $\left\{q_{n}^{x}: n \in \mathbb{N}\right\},\left\{q_{n}^{y}: n \in \mathbb{N}\right\},\left\{q_{n}^{a}: n \in \mathbb{N}\right\} \subset \mathbb{Q}$ with $a=\lim _{n \rightarrow \infty} F\left(b, q_{n}^{a} x_{0}\right), F(b, x)=\lim _{n \rightarrow \infty} F\left(b, q_{n}^{x} x_{0}\right)$, and $F(b, y)=$ $\lim _{n \rightarrow \infty} F\left(b, q_{n}^{y} x_{0}\right)$ (we construct these sequences e.g. as in the proof of Lemma 1). According to Lemmas $2-4$ and $\left(1^{\prime}\right)$,

$$
\begin{aligned}
F(b, x+y) & =F(F(b, x), y)=\lim _{n \rightarrow \infty} F\left(F(b, x), q_{n}^{y} x_{0}\right) \\
& =\lim _{n \rightarrow \infty}\left(\lim _{k \rightarrow \infty} F\left(F\left(b, q_{k}^{x} x_{0}\right), q_{n}^{y} x_{0}\right)\right. \\
& =\lim _{n \rightarrow \infty}\left(\lim _{k \rightarrow \infty} F\left(b,\left(q_{k}^{x}+q_{n}^{y}\right) x_{0}\right)\right)=\lim _{n \rightarrow \infty} F\left(b,\left(q_{n}^{x}+q_{n}^{y}\right) x_{0}\right) .
\end{aligned}
$$

Thus

$$
\begin{aligned}
c_{t}(x+y) & =f_{t}(F(b, x+y))=t \lim _{n \rightarrow \infty}\left(q_{n}^{x}+q_{n}^{y}\right) \\
& =f_{t}(F(b, x))+f_{t}(F(b, y))=c_{t}(x)+c_{t}(y) .
\end{aligned}
$$

Consequently, $c_{t}$ is additive. Further, by Lemmas 2-4,

$$
\begin{aligned}
F(a, x) & =\lim _{k \rightarrow \infty} F\left(a, q_{k}^{x} x_{0}\right)=\lim _{k \rightarrow \infty}\left(\lim _{n \rightarrow \infty} F\left(F\left(b, q_{n}^{a} x_{0}\right), q_{k}^{x} x_{0}\right)\right) \\
& =\lim _{n \rightarrow \infty} F\left(b,\left(q_{n}^{a}+q_{n}^{x}\right) x_{0}\right) .
\end{aligned}
$$

Hence

$$
f_{t}(F(a, x))=t \lim _{n \rightarrow \infty}\left(q_{n}^{a}+q_{n}^{x}\right)=f_{t}(a)+f_{t}(F(b, x))=f_{t}(a)+c_{t}(x) .
$$


Since, in view of Lemma $1, f_{t}$ is one-to-one, this implies (9).

To complete the proof, note that, by $(4), F(b, 0)<F\left(b, x_{0}\right)$. Thus, on account of Lemma 1 , for $t>0$,

$$
0=c_{t}(0)=f_{t}(F(b, 0))<f_{t}\left(F\left(b, x_{0}\right)\right)=c_{t}\left(x_{0}\right),
$$

and for $t<0$,

$$
0=c_{t}(0)=f_{t}(F(b, 0))>f_{t}\left(F\left(b, x_{0}\right)\right)=c_{t}\left(x_{0}\right) .
$$

So, we have proved Lemma 5 .

Now, we are in a position to prove the following

THEOREM 1. Assume that $(G,+$ ) is a group (not necessarily commutative) and has a uniquely divisible subgroup $H$. Let $b \in M$ and $x_{0} \in H \backslash\{0\}$. Then a function $F: M \times G \rightarrow M$ satisfies conditions $\left(1^{\prime}\right),(3)$, (4), and (6)-(8) if and only if there exist a non-empty set $T \subset \mathbb{R}$, an additive function $c: G \rightarrow \mathbb{R}, c\left(x_{0}\right)>0\left(c\left(x_{0}\right)<0\right.$ resp.), and an increasing (decreasing resp.) bijection $f: M \rightarrow K$, where $K=T+c(G)$, such that (2) holds for every $x \in G$ and $a \in M$. Furthermore, every function $F: M \times G \rightarrow M$ of the form (2) is a solution of equation (1).

Proof. Assume that $F: M \times G \rightarrow M$ satisfies conditions $\left(1^{\prime}\right),(3),(4)$, and (6)-(8). Fix $t \in \mathbb{R}, t>0$ ( $t<0$ resp.). Then, according to Lemmas 1 and 5 , the function $f_{t}: M \rightarrow \mathbb{R}$ given by (5) is strictly increasing (decreasing resp.), the function $c_{t}: G \rightarrow \mathbb{R}, c_{t}(z)=f_{t}(F(b, z))$ for $z \in G$, is additive, $c_{t}\left(x_{0}\right)>0\left(c_{t}\left(x_{0}\right)<0\right.$ resp. $)$, and $(9)$ holds. Thus it suffices to put $T=$ $f_{t}(M)$.

Now, assume that $F: M \times G \rightarrow M$ is of the form (2). It is easy to check that $F$ is a solution of equation (1) and satisfies (8). Conditions (4) and (7) result from the fact that $f$ is increasing (decreasing resp.) and $c\left(x_{0}\right)>0$ $\left(c\left(x_{0}\right)<0\right.$ resp.). Further, since $c$ is additive and $H$ is uniquely divisible, $c\left(q x_{0}\right)=q c\left(x_{0}\right)$ for $q \in \mathbb{Q}$. Thus the sets $c(G)$ and $K$ are dense in $\mathbb{R}$, which means that $s+c(G)$ and $K+r$ are dense in $K$ for every $s \in K$ and $r \in c(G)$. Consequently, by (2), conditions (3) and (6) hold, because $f$ is a monotonic bijection. This completes the proof.

From Theorem 1 we obtain the following corollary, which gives a solution of the problem of J. Tabor.

Corollary 1. Suppose that $G$ is a uniquely divisible group. Then a function $F: M \times G \rightarrow M$ satisfies equation (1) and there exist $b \in M$, $x_{0} \in G \backslash\{0\}$ such that conditions (3), (4), and (6)-(8) are valid if and only if there exist a non-empty set $T \subset \mathbb{R}$, an additive function $c: G \rightarrow \mathbb{R}$, $c(G) \neq\{0\}$, and an increasing bijection $f: M \rightarrow K$, where $K=T+c(G)$, such that (2) holds for $x \in G$ and $a \in M$. 
Proof. It suffices to note that $c(G) \neq\{0\}$ iff there is $x_{0} \in G$ with $c\left(x_{0}\right)>0$.

Remark 1. The assumption of Theorem 1 that $G$ is a group can be weakened. Namely, it suffices to suppose that $G$ is a groupoid (i.e. a nonempty set endowed with a binary operation) and has a subgroupoid $H$ which is a uniquely divisible group.

The representation (2) of a solution $F: M \times G \rightarrow M$ of equation (1) is not unique:

Proposition 1. Suppose that $G$ is as in Theorem $1, c_{1}, c_{2}: G \rightarrow \mathbb{R}$ are additive functions such that $c_{i}(H) \neq\{0\}$ for $i=1,2, T_{1}, T_{2} \subset \mathbb{R}$ are non-empty sets, $f_{i}: M \rightarrow K_{i}$, where $K_{i}=T_{i}+c_{i}(G)$, for $i=1,2$ are monotonic bijections, and

$$
F_{i}(a, x)=f_{i}^{-1}\left(f_{i}(a)+c_{i}(x)\right) \quad \text { for } x \in G, a \in M, i=1,2 .
$$

Then $F_{1}=F_{2}$ iff there exist $u, v \in \mathbb{R}, u \neq 0$, such that $f_{1}(a)=u f_{2}(a)+v$ for $a \in M$ and $c_{1}(x)=u c_{2}(x)$ for $x \in G$.

Proof. First suppose that there are $u, v \in \mathbb{R}, u \neq 0$, with $f_{1}=u f_{2}+v$ and $c_{1}=u c_{2}$. Then, for every $a \in M$ and $x \in G$,

$$
\begin{aligned}
F_{1}(a, x) & =f_{1}^{-1}\left(f_{1}(a)+c_{1}(x)\right)=f_{1}^{-1}\left(u\left(f_{2}(a)+c_{2}(x)\right)+v\right) \\
& =f_{1}^{-1}\left(u f_{2}\left(F_{2}(a, x)\right)+v\right)=F_{2}(a, x) .
\end{aligned}
$$

Now, assume that $F_{1}=F_{2}$. Fix $t \in \mathbb{R}, t>0, b \in M$, and $x_{0} \in H \backslash\{0\}$ with $c_{1}\left(x_{0}\right)>0$. Then, by $(10)$, we also have $c_{2}\left(x_{0}\right) \neq 0$. Put $F=F_{1}$. According to Theorem 1, (3) and (4) hold. Thus, by Lemma 1, the function $f_{t}: M \rightarrow \mathbb{R}$ given by (5) is well defined.

Fix $i \in\{1,2\}$ and $a \in M$. Let $\left\{q_{n}^{a}: n \in \mathbb{N}\right\} \subset \mathbb{Q}$ be a strictly increasing sequence with $a=\lim _{n \rightarrow \infty} F\left(b, q_{n}^{a} x_{0}\right)$. Note that $f_{i}(F(b, x))=f_{i}(b)+c_{i}(x)$ for $x \in G$. Thus the set $\left\{f_{i}\left(F\left(b, p x_{0}\right)\right): p \in \mathbb{Q}\right\}$ is dense in $\mathbb{R}$ and the sequence $\left\{f_{i}\left(F\left(b, q_{n}^{a} x_{0}\right)\right): n \in \mathbb{N}\right\}$ is strictly monotonic. Moreover, by (4), for each $p \in \mathbb{Q}$ with $F\left(b, p x_{0}\right)<a$, there is $n_{0} \in \mathbb{N}$ such that $F\left(b, p x_{0}\right)<$ $F\left(b, q_{n}^{a} x_{0}\right)<a$ for $n \in \mathbb{N}, n>n_{0}$. Consequently, since $f_{i}$ is monotonic, $f_{i}(a)=\lim _{n \rightarrow \infty} f_{i}\left(F\left(b, q_{n}^{a} x_{0}\right)\right)$. Hence, for each $a \in M$,

$$
\begin{aligned}
f_{i}(a) & =\lim _{n \rightarrow \infty}\left(f_{i}(b)+c_{i}\left(q_{n}^{a} x_{0}\right)\right)=f_{i}(b)+c_{i}\left(x_{0}\right) \lim _{n \rightarrow \infty} q_{n}^{a} \\
& =f_{i}(b)+t^{-1} c_{i}\left(x_{0}\right) f_{t}(a)
\end{aligned}
$$

and, for each $z \in G$,

$$
f_{i}(b)+c_{i}(z)=f_{i}(F(b, z))=f_{i}(b)+t^{-1} c_{i}\left(x_{0}\right) f_{t}(F(b, z)) .
$$

So, we have proved that there are $u_{1}, u_{2}, v_{1}, v_{2} \in \mathbb{R}, u_{1} u_{2} \neq 0$, with $f_{t}(a)=$ 
$u_{i} f_{i}(a)+v_{i}$ and $f_{t}(F(b, z))=u_{i} c_{i}(z)$ for $a \in M, z \in G, i=1,2$. Hence it suffices to put $u=u_{2} u_{1}^{-1}$ and $v=u_{1}^{-1}\left(v_{2}-v_{1}\right)$. This ends the proof.

Remark 2. Let $x_{0} \in H \backslash\{0\}$ and $b \in M$. A function $F: M \times G \rightarrow M$ satisfies equation (1) and condition (4) iff the function $F_{0}: M \times G \rightarrow M$, $F_{0}(a, x)=F(a,-x)$ for $a \in M, x \in G$, satisfies (1) and

$$
F_{0}\left(b, p x_{0}\right)>F_{0}\left(b, q x_{0}\right) \quad \text { for } p, q \in \mathbb{Q}, p<q .
$$

Thus, from Theorem 1 we can also get a description of solutions of (1) satisfying conditions (3), (6), (7), and $\left(4^{\prime}\right)$.

Remark 3. Suppose that $x_{0} \in H \backslash\{0\}, b \in M$, and a function $F$ : $M \times G \rightarrow M$ satisfies (4), equation (1), and the condition

$$
F(a, x)<F(c, x) \quad \text { for } a, c \in M, a>c, x \in G .
$$

Fix $p, q \in \mathbb{Q}$ with $p<q$. Then, by $(4), F\left(b, p x_{0}\right)<F\left(b, q x_{0}\right)$. Thus, on account of $\left(7^{\prime}\right)$,

$$
F\left(b,(p+1) x_{0}\right)=F\left(F\left(b, p x_{0}\right), x_{0}\right)>F\left(F\left(b, q x_{0}\right), x_{0}\right)=F\left(b,(q+1) x_{0}\right),
$$

which, in view of (4), means that $p+1>q+1$. This gives a contradiction. Consequently, there are no solutions of (1) satisfying conditions (4) and $\left(7^{\prime}\right)$, and similarly for $\left(4^{\prime}\right)$ and $\left(7^{\prime}\right)$ according to Remark 2 .

Remark 4. Suppose that $H$ is endowed with a linear order such that, for every $x, y, z \in H$,

$$
x<y \quad \text { iff } \quad z+x<z+y \text { and } x+z<y+z .
$$

Let $x_{0} \in H, x_{0}>0$, and $b \in M$. Then every function $F: M \times G \rightarrow M$ such that $F(b, x)<F(b, y)$ for every $x, y \in H, x<y$, also satisfies (4).

In fact, let $p, q \in \mathbb{Q}, p<q$. Then there are $j, k, m, n \in \mathbb{Z}, k>0, n>0$, with $p=j k^{-1}$ and $q=m n^{-1}$. Note that $j n<k m$. Thus $k n p x_{0}=n j x_{0}<$ $k m x_{0}=k n q x_{0}$, whence $p x_{0}<q x_{0}$. Hence $F\left(b, p x_{0}\right)<F\left(b, q x_{0}\right)$.

\section{References}

[1] U. Abel, Sur les groupes d'itération monotones, Publ. Math. Debrecen 29 (1982), 65-71.

[2] J. Aczél, L. Kalmár et J. G. Mikusiński, Sur l'équation de translation, Studia Math. 12 (1951), 112-116.

[3] A. Grzegorczyk and J. Tabor, Monotonic solutions of the translation equation, Ann. Polon. Math. 43 (1983), 253-260. 
[4] J. Tabor, Characterization of mixed iteration groups, to appear.

Institute of Mathematics

Pedagogical University of Rzeszów

Rejtana 16A

35-310 Rzeszów, Poland

Reçu par la Rédaction le 16.11.1991

Révisé le 8.11.1995 